\title{
Understanding the Role of Knowledge Management during the ERP Implementation Lifecycle: Preliminary Research Findings Relevant to Emerging Economies
}

\author{
Anjali Ramburn, Lisa F. Seymour, and Avinaash Gopaul \\ Information Systems Department, University of Cape Town, Private Bag, \\ Rondebosch 7700, South Africa \\ \{Anjali.Ramburngopaul, Li.sa. Seymour\}@uct.ac.za
}

\begin{abstract}
This work in progress paper presents a preliminary analysis on the challenges of knowledge management (KM) experienced in the ERP implementation phase. This paper is an integral section of an ongoing research focusing on the role of KM during the ERP implementation lifecycle in both large and medium organizations in South Africa. One of the key research objectives is to investigate the core challenges of $\mathrm{KM}$ in large and medium organizations in South Africa. A review of the existing literature reveals a lack of comprehensive KM research during the different ERP implementation phases and particularly, in emerging economies. Initial findings include lack of process, technical and project knowledge as key challenges. Other concerns include poor understanding of the need for change, lack of contextualization and management support. This paper closes some of the identified research gaps in this area and should benefit large organizations in the South African economy.
\end{abstract}

Keywords: Knowledge Management, ERP Implementation, ERP Implementation Phase, Emerging Economy.

\section{Introduction}

\subsection{Background and Context}

Organizations are continuously facing challenges, causing them to rethink and adapt their strategies, structures, goals, processes and technologies in order to remain competitive [1], [2]. Many large organizations are now dependent on ERP systems for their daily operations. An increasing number of organizations are investing in ERP systems in South Africa. There have been many implementations in the South African public sector such as the SAP implementations at the City of Cape Town and Tshwane Metropolitan Council. The implementation process is however described as costly, complex and risky whereby firms are not able to derive benefits of the systems despite huge investments. Half of all ERP implementations fail to meet the adopting organizations' expectations [4]. This has been attributed to the disruptive and threatening nature of ERP implementations [5], [6]. This process however can be less challenging and more effective through proper use of knowledge management (KM) 
throughout the ERP lifecycle phases. Managing ERP systems knowledge has been identified as a critical success factor and as a key driver of ERP success [7]. An ERP implementation is a dynamic continuous improvement process and "a key methodology supporting ERP continuous improvement would be knowledge management" [8].

\subsection{Research Problem, Objective and Scope}

There has been very little work conducted to date that assesses the practices and techniques employed to effectively explain the impact of KM in the ERP systems lifecycle [9], [10]. Current research in the context of $\mathrm{KM}$ focuses mostly on knowledge sharing and integration challenges during the actual ERP adoption process, offering only a static perspective of KM and ERP implementation [11], [12], [13]. A number of organizations see the ERP GO Live as the end of the cycle, and very little emphasis has been given to the post implementation phases.

This research seeks to explore the ERP implementation life cycle from a KM perspective within a South African context and aims at providing a comprehensive understanding of the role of KM practices during the ERP implementation lifecycle. One of the key objectives is to investigate the KM challenges faced by organizations while implementing ERP systems. This paper therefore presents the findings of KM challenges experienced during the implementation phase of an ERP system. It should be noted that the results, discussed in this paper, are an interpretation of the initial findings which is still under review. This analysis will be further developed and elaborated in the subsequent research phases.

\section{$2 \quad$ Literature Review}

\subsection{Enterprise Resource Planning Systems}

An ERP system can be defined as "an information system that enables the integration of transaction-based data and business processes within and across functional areas in an enterprise" [9]. Some of the key enterprise functions that ERP systems support include supply chain management, inventory control, sales, manufacturing scheduling, customer relationship management, financial and cost management and human resources [10], [14]. Despite the cost intensive, lengthy and risky process, the rate of implementation of ERP systems has increased over the years. Most of the large multinational organizations have already adopted ERPs as their de facto standard with the aim of increasing productivity, efficiency and organizational competitiveness [15].

\subsection{Role and Challenges of Knowledge Management}

$\mathrm{KM}$ is defined as an on-going process where knowledge is created, shared, transferred to those who need it, and made available for future use in the organization [16]. Effective use of $\mathrm{KM}$ in ERP implementation has the potential to improve organizational efficiency during the ERP implementation process [7]. Successful 
transfer of knowledge between different ERP implementation stakeholders such as the client, implementation partner and vendor is important for the successful implementation an ERP system.

Use of KM activities during the ERP implementation phase ensures reduced implementation costs, improved user satisfaction as well as strategic and competitive business advantages through effective product and process innovation during use of ERP [10]. Organizations should therefore be aware of and identify the knowledge requirement for any implementation. However, a number of challenges hindering the proper diffusion of KM activities during the ERP implementation phase have been highlighted. The following potential knowledge barriers have been identified by [15].

Knowledge Is Embedded in Complex Organizational Processes. ERP systems' capabilities and functionalities span across different departments involving many internal and external users, leading to a diversity of interest and competencies in specific knowledge areas. A key challenge is to overcome any conflicting interest in order to integrate knowledge in order to promote standardization and transparency.

Knowledge Is Embedded in Legacy Systems. Users are reluctant to use the new systems, constantly comparing the capabilities of the new systems to legacy systems. This is a prevalent mindset which needs to be anticipated and [15] suggest the ERP system looks outwardly similar to the legacy system through customization. This can be achieved by "integrating knowledge through mapping of the information, processes, and routines of the legacy system into the ERP systems with the use of conversion templates" [15].

Knowledge Is Embedded in Externally Based Processes. ERP systems link external systems to internal ones, as a result external knowledge from suppliers and consultants needs to be integrated in the system. This can be a tedious process and the implementation team needs to ensure that essential knowledge is integrated from the initial implementation phases through personal and working relationships.

\subsection{Gaps in the Literature}

The literature review indicates that most of the studies performed in the context of KM and ERP implementation offer a one dimensional static view of the actual ERP adoption phases without emphasizing the overall dynamic nature of ERP systems. Furthermore, previous studies have failed to provide a holistic view of the challenges, importance, different dimensions and best practices of KM during the whole ERP implementation cycle.

\section{$3 \quad$ Research Method}

\subsection{Research Paradigm and Approach}

This research employs an interpretive epistemology which is ideal for this research as this study focuses on theory building, where the ERP implementation challenges 
faced by organizations are explored using a knowledge perspective [17]. A qualitative research method is deemed suitable as opposed to a quantitative one, as qualitative research emphasizes on non- positivist, non-linear and cyclical forms of research, allowing the scientist to gain new insights of the research area through each iteration whilst aiming to provide a better understanding to the social world [18], [21].

Grounded theory seems particularly applicable in the current context as there has been no exhaustive analysis on, barriers, dimensions and role of KM focusing on the whole ERP implementation life cycle in organizations. Grounded theory used in this research is an "inductive, theory-discovering methodology that allows the researcher to develop a theoretical account of the general features of a topic, while simultaneously grounding the account in empirical observations of data" [19], [20].

Semi-Structured interviews targeting different ERP implementation stakeholders are being conducted in an organization currently in their ERP implementation phase. The aim is to interview as many participants as possible until theoretical saturation is achieved. Approval for this research has been obtained from the University of Cape Town's ethical committee. Participants have been asked to sign a voluntary participant consent form and their anonymity has been assured.

All the interviews have been recorded and transcribed. Iterative analysis of the collected data has enabled the researcher to understand and investigate the main research problems posed. The transcripts of the interviews have been read a number of times to identify, conceptualise, and categorise emerging themes.

\subsection{Case Description}

This section provides a brief overview of the case organization. Founded in 1923, the company has a number of branches throughout South Africa, employing over 39000 people. The organization is currently launching the SAP Project Portfolio Management module throughout its different branches across the country. Currently in the implementation stage, an organization wide initial training, involving the employees, has already been conducted. The interviews have been carried out in one of organization's division in Cape Town and purposive sampling has been used to select the interviewees. All the chosen participants had been through the training and were impacted by the SAP implementation process.

\section{$4 \quad$ Preliminary Findings}

Preliminary research findings indicate several challenges with regards to KM in the ERP implementation phase. Most of the barriers identified were either directly or indirectly related to the inadequacies and inefficiencies of knowledge transfer. The section below provides a comprehensive account of the major challenges that have been identified. 


\subsection{Knowledge Management Challenges}

Trainer's Lack of Process Knowledge. Interviewees mentioned the training provided was inadequate in various ways. The trainers were not knowledgeable enough; they lacked key SAP skills and did not understand the process from the users' perspective. Since none of the trainers had any experience as end users of the system, there were some inconsistencies in their understanding of the new system from a user perspective. Ownership of roles and tasks were not clearly defined. They also lacked the expertise to engage with the different problems that surfaced during the training and there was no clarification on the information and process flow between the different departments and the individuals as per their role definition. "However what makes it difficult is that the trainers do not work with the project. They do not know the process entirely and are not aware of what is happening in the background, they only collect data."

Trainer's Lack of Technical Knowledge. The technical knowledge and qualification of the trainers were put into question. The trainers were the admin support technicians who are experts in the current system the interviewees use but did not have enough expertise to deal with the upcoming ERP system. "I think they did not know the system themselves, I had been in training with them for the current program we use and they were totally 100\% clued up. You could have asked them anything, they had the answers."

Interviewees' Lack of Technical Knowledge. Interviewees also struggled with use and understanding of the ERP system. They found the user interface and navigation increasingly complex as opposed to their existing system. As a result, they were overcome with frustration and they did not see the importance of the training. "I have not used the system before, so I do not understand it. We struggled with the complexity of the system. The number of steps we had to do made it worse. No one understood what and why we were doing most of the steps."

Lack of Knowledge on Need for Change. The interviewees did not understand the benefits of using SAP from a strategic perspective. They questioned the implementation of the new system as they felt their previous system could do everything they needed it to. They had never felt the need for a new system.

Lack of Project knowledge. Interviewees were unaware of the clear project objectives, milestones and deployment activities. The interviewees did not have any information regarding the status of the project activities. They were only aware of the fact that they had to be trained in SAP as this would be their new system in the future but did not exactly know by when they were required to start using the system. Some of them believed they were not near the implementation stage, and the training was only a pilot activity to test whether they were ready for implementation. However, others hoped that the implementation had been cancelled due to the number of problems experienced in the training sessions. 
Poor Project Configuration Knowledge. Another key concern voiced related to the complexity of the ERP system as opposed to the existing system the participants are using. They have been working with the current system for a number of years and believed it operated in the most logical way, the same way as to how their minds would function. On the other hand, the ERP system was perceived as complex, the number of steps required to perform for a task seem to have increased drastically. This may be attributed to the lack of system configuration knowledge which could have been essential in substantially decreasing the number of steps required to perform a particular task.

Lack of Knowledge on Management Initiatives. The interviewees felt they did not have to use or understand the system until they got the 'go ahead' from top and middle management. Interviews indicated that top and middle management had not supported the initiative as yet. Interviewees had received no information or communication on planning, adoption and deployment of the new system from management; hence they showed no commitment towards using the new system.

Lack of Knowledge on Change Management Initiatives. Managing change is arguably one of the primary concerns of ERP implementation. The analysis show the lack of importance attributed to this area. Lack of proper communication channels and planning coupled with the absence of change management initiatives resulted in employees' confusion, instability and resistance as shown by quotes below. "We should not have used SAP at all, they should scrap it...If someone new came and asked me whether they should go for the training, I would tell them, try your best to get out of it."

Knowledge Dump (Information overload). Information overload was another identified challenge. The training included people from different departments who are associated with different aspects of the process. As a result, the trainers covered various tasks related to various processes in one training session as opposed to focusing on the specific processes that the interviewees understood and were involved with. The participants got confused with regards to their role definition and the ownership of the different activities. The trainers were unable to clear this confusion. This caused a certain level of panic amongst the group; subsequently they lost interest in the training and attributed it as an unproductive process.

Poor Contextualization of Knowledge. Another concern raised was with reference to the lack of customization of the training materials and exercises used resulting in a poor focus on local context. Interviewees could not relate to the training examples given as they were based on the process flow from a different suburb. Interviewees said each suburb has its own way of operating and has unique terms and terminologies. The fact that the examples used came from Johannesburg and not from Cape Town made it harder for the interviewees to understand the overall process. "The examples they used were from Joburg, so they work in a different way to us. The 
examples should have been customised to how we work in order for us to better understand the process."

\section{Conclusions and Implications}

This paper reports on the preliminary findings based on the implementation activities of an ERP system in a large engineering company in Cape Town. The findings of this study show a number of intra-organizational barriers to efficient knowledge transfer. Inadequate training, lack of technical and project knowledge, lack of management support and change management initiatives have been cited as the major KM challenges. Other fundamental KM challenges include process knowledge, customization and contextualization of knowledge. Seemingly, in a large organization with multiple branches throughout South Africa, understanding the process, contextualization and customization of the training content from the users' perspective is a key aspect to consider during an ERP implementation process.

This research is still ongoing and the subsequent research phases focus on providing a holistic view of the role, different dimensions and best practices of KM during the entire ERP implementation cycle. Upon completion, this research will be of immediate benefit to both academics and practitioners.

From an academic perspective, this study will explore the whole ERP implementation lifecycle from a KM perspective, hence contributing to the existing body of knowledge in this area by attempting to offer a better explanation of the existing theories and frameworks. Since there has not been any study that looked at the entire lifecycle of ERP implementation through a KM perspective in South Africa, this research is unique in nature and is expected to break some new ground in South Africa, aiming to provide an advancement of knowledge in this particular field. Through a practical lens, this research should be of immediate benefit to large and medium organizations. The results of this study can also be useful and applicable to international companies with global user bases.

\section{References}

1. Bhatti, T.R.: Critical Success Factors for the Implementation of Enterprise Resource Planning (ERP): Empirical Validation. In: 2nd International Conference on Innovation in Information Technology, Zayed University, College of Business, Dubai, UAE (2005)

2. Holland, C.P., Light, B.: A critical success factors model for ERP implementation. IEEE Software 16(3), 30-36 (1999)

3. Seethamraju, R., Seethamraju, J.: Adoption of ERPs in a Medium-sized Enterprise - A Case Study. In: 19th Australasian Conference on Information Systems Adoption of ERPs for Medium-sized Enterprise, Christchurch, December 3-5 (2008)

4. Jasperson, J.S., Carter, P.E., Zmud, R.W.: Conceptualization of Post-Adoptive Behaviours Associated with Information Technology Enabled Work Systems. MIS Quarterly 29(3), 525-567 (2005)

5. Zorn, T.E.: The Emotionality of Information and Communication Technology Implementation. Journal of Communication Management 7(2), 160-171 (2002) 
6. Robey, D., Ross, J., Boudreau, M.: Learning to Implement Enterprise Systems: An Exploratory Study of the Dialectics of Change. Journal of Management Information Systems 19(1), 17-46 (2002)

7. Leknes, J., Munkvold, B.E.: The role of knowledge management in ERP implementation: a case study in Aker Kvaerner. In: 14th European Conference on Information Systems (ECIS 2006), Göteborg, Sweden, June 12-14 (2005)

8. McGinnis, T.C., Huang, Z.: Incorporating of Knowledge Management into ERP continuous improvement: A research framework. Issues in Information Systems 2, 612-618 (2004)

9. Parry, G., Graves, A.: The importance of knowledge management for ERP systems. International Journal of Logistics Research and Applications 11(6), 427-441 (2008)

10. Sedera, D., Gable, G., Chan, T.: Knowledge Management for ERP success. In: 7th Pacific Asia Conference on Information Systems, Adelaide, South Australia, July 10-13 (2004)

11. Suraweera, T., Remus, U., Wakerley, S.: Dynamics of Knowledge Leverage in ERP Implementation. In: 18th Australasian Conference on Information Systems, Toowoomba, Australia, December 5-7 (2007)

12. Gable, G.: The enterprise system lifecycle: through a knowledge management lens. Strategic Change 14, 255-263 (2005)

13. Markus, M.L.: Towards a Theory of Knowledge Reuse: Types of Knowledge Reuse Situations and Factors in Reuse Success. Journal of Management Information Systems 18(1), 57-93 (2001)

14. Soffer, P., Golany, B., Dori, D.: ERP modeling: a comprehensive approach. Journal of Information Systems 28, 673-690 (2002)

15. Pan, S.L., Huang, J.C., Newell, S., Cheung, A.W.K.: Knowledge Integration as a key problem in an ERP Implementation. In: 22nd International Conference on Information Systems, December 16-19, New Orleans, Louisiana (2004)

16. Chan, R.: Knowledge management for implementing ERP in SMEs. In: 3rd Annual SAP Asia Pacific Institutes of Higher Learning Forum Maximizing the Synergy Between Teaching, Research and Business, Singapore, November 1-2 (1999)

17. Walsham, G.: Interpretive case studies in IS research: Nature and method. European Journal of Information Systems 4, 74-81 (1995)

18. Leedy, P.D.: Practical research: planning and design, 6th edn. Prentice-Hall, Upper Saddle River (1997)

19. Glaser, B.G., Strauss, A.L.: The Discovery of Grounded Theory: Strategies for Qualitative Research, Chicago. Aldine Publishing Company, Hawthorne (1967)

20. Orlikowski, W.J.: CASE tools as organisational change: Investigating incremental and radical changes in systems development. MIS Quarterly 17, 309-340 (1993)

21. Strauss, A., Corbin, J.: Basics of Qualitative Research: Grounded Theory Procedure and Techniques. Sage, Newbury Park (1990)

22. Fang, L., Patrecia, S.: Critical Success Factors in ERP Implementation. Master thesis. Jànkàping International Business School (2005)

23. Huang, Z.: A compilation research of ERP implementation Critical Success Factors. Issues in Information Systems 11(1), 507-512 (2010)

24. Somers, T.M., Nelson, K.: The impact of critical success factors across the stages of enterprise resource planning implementations. In: 34th Hawaii International Conference on Systems Sciences, Maui (2001) 